\title{
Besicovitch via Baire
}

\author{
by \\ T. W. KÖRNER (Cambridge)
}

\begin{abstract}
We construct various Besicovitch sets using Baire category arguments.
\end{abstract}
1. Introduction. A Besicovitch set is a compact subset $E$ of $\mathbb{R}^{n}$ of Lebesgue measure zero containing line segments of length 1 in every direction $[n \geq 2]$. (Formally, if $\mathbf{u}$ is a unit vector, there exists an $\mathbf{x}$ such that $\mathbf{x}+\lambda \mathbf{u} \in E$ for all $0 \leq \lambda \leq 1$.) The first example of such a set was given by Besicovitch in [2] and many different examples have been given since. Besicovitch sets turned out to be unexpectedly important in harmonic analysis (see Chapter X of [7]).

In Section 2, I give a Baire category argument for the existence of Besicovitch sets. I hope that some readers will find it an attractive alternative to the standard proofs.

In the next section (Section 3), I derive some further generic properties of our sets. It turns out that our sets are (quasi-always) more irregular than those produced by the classical argument set out in Chapter X of [7], by the remarkable method of Kahane [6] and by the random constructions of Alexander [1]. (Of course, this makes them less rather than more interesting for the purposes of harmonic analysis.)

The paper of Alexander [1] uses the fundamental theorem of Besicovitch [3] which states that, if an irregular set $S$ in $\mathbb{R}^{2}$ has finite linear measure, then $S$ has an orthogonal projection $S_{\theta}$ of zero linear measure for almost all directions $\theta$. If we take $S=E \times E$ where $E$ is a Cantor set with ratio of dissection $1 / 4$, then this shows that the set $E+\lambda E$ considered in [6] will have zero linear measure for almost every value of $\lambda$ and so the set considered by Kahane does, indeed, have area 0. Kahane informs me that the argument used in his paper to obtain this result was incorrect and that he owes the correct argument just given to Alexander.

2000 Mathematics Subject Classification: Primary 28A75.

Key words and phrases: Besicovitch set, Baire category, Hausdorff metric. 
In the final section (Section 4), I develop the method of Section 2 slightly to obtain the following result which I believe to be new.

TheOREm 1.1. Let $1>a>0$ and let $n$ be an integer with $n \geq 2$. There exists a collection $\mathcal{L}$ of line segments in $\mathbb{R}^{n}$ each of length at least $2-$ a such that

(i) There is a line segment $l \in \mathcal{L}$ in any given direction.

(ii) $\bigcup_{l \in \mathcal{L}} l$ is a closed set lying in the ball with centre the origin and radius 1 .

(iii) If $\widehat{l}$ is any line then we can find $\mathcal{L}_{0}$ with $\mathcal{L}_{0} \subseteq \mathcal{L}$ and $\mathcal{L} \backslash \mathcal{L}_{0}$ containing at most one element such that $\bigcup_{l \in \mathcal{L}_{0}} l \cap \widehat{l}$ has zero linear Lebesgue measure.

2. A Besicovitch set. We shall work in $\mathbb{R}^{2}$ but the argument is easily extended to $\mathbb{R}^{n}$. Consider the collection $\mathcal{K}$ of non-empty compact subsets of $\mathbb{R}^{2}$. If we define

$$
\varrho(E, F)=\sup _{\mathbf{e} \in E} \inf _{\mathbf{f} \in F}\|\mathbf{e}-\mathbf{f}\|, \quad d(E, F)=\varrho(E, F)+\varrho(F, E)
$$

for any $E, F \in \mathcal{K}$ then it is easy to check that $d$ is a complete metric (the Hausdorff metric; see Section 28 of [5]) on $\mathcal{K}$.

We study the following set.

Definition 2.1. $\mathcal{P}$ is the collection of all closed subsets $P$ of the rectangle $[-1,1] \times[0,1]$ with the following properties:

(i) $P$ is the union of line segments joining points of the form $\left(x_{1}, 0\right)$ to points of the form $\left(x_{2}, 1\right)$ with $x_{1}, x_{2} \in[-1,1]$.

(ii) If $|v| \leq 1 / 2$ then we can find $x_{1}$ and $x_{2}$ with $x_{2}-x_{1}=v$ and $x_{1}, x_{2} \in[-1,1]$ such that the line segment joining $\left(x_{1}, 0\right)$ to $\left(x_{2}, 1\right)$ lies in $P$.

Lemma 2.2. $\mathcal{P}$ is a closed subset of $(\mathcal{K}, d)$.

Proof. Suppose $P_{n} \in \mathcal{P}, K \in \mathcal{K}$ and $d\left(P_{n}, K\right) \rightarrow 0$. We first seek to show that $K$ satisfies property (i) in Definition 2.1. To this end, suppose that $\mathbf{k} \in K$. By definition, we can find $\mathbf{p}_{n} \in P_{n}$ with $\left\|\mathbf{p}_{n}-\mathbf{k}\right\| \rightarrow 0$ as $n \rightarrow \infty$. Since $P_{n}$ has property (i), we can find $x_{1, n}, x_{2, n} \in[-1,1]$ such that the line segment $l_{n}$ joining $\left(x_{1, n}, 0\right)$ to $\left(x_{2, n}, 1\right)$ contains $\mathbf{p}_{n}$. By the compactness of $[-1,1]^{2}$, we can find an integer sequence $n(j) \rightarrow \infty$ and $x_{1}, x_{2} \in[-1,1]$ such that $x_{1, n(j)} \rightarrow x_{1}$ and $x_{2, n(j)} \rightarrow x_{2}$ as $j \rightarrow \infty$. If we denote the line segment joining $\left(x_{1}, 0\right)$ to $\left(x_{2}, 0\right)$ by $l$ then $d\left(l_{n(j)}, l\right) \rightarrow 0$ as $j \rightarrow \infty$. It follows that $l \subseteq K$ and $\mathbf{k} \in l$. We have established that $K$ has property (i).

The proof that $K$ has property (ii) is similar.

From now on we consider the space $(\mathcal{P}, d)$ with the restriction metric. By Lemma $2.2,(\mathcal{P}, d)$ is complete. We can now state our first theorem. 
TheOREM 2.3. The set of $P \in \mathcal{P}$ of Lebesgue measure zero is of second category in $(\mathcal{P}, d)$.

Since a set of second category in a complete metric space is non-empty, it follows that there exists a set $P_{0} \in \mathcal{P}$ of Lebesgue measure zero. By part (ii) of Definition 2.1, $P_{0}$ contains line segments of length at least 1 in every direction making an angle of absolute value less than or equal to $\pi / 6$ with the $y$-axis. If we take the union of three copies of $P_{0}$ rotated through $0, \pi / 3$ and $2 \pi / 3$ the result will be a Besicovitch set.

The key to our proof of Theorem 2.3 is the following lemma.

LEMMA 2.4. If $v \in[0,1]$ and $\varepsilon>0$, write $\mathcal{P}(v, \varepsilon)$ for the set of $P \in \mathcal{P}$ with the following property. There exist a finite collection of rectangles $R_{1}, \ldots, R_{N}$, say, with sides parallel to the axes, such that, if $y \in[0,1] \cap$ $[v-\varepsilon, v+\varepsilon]$, then

$$
\{x:(x, y) \in P\} \subseteq\left\{x:(x, y) \in \bigcup_{j=1}^{N} R_{j}\right\}
$$

and the total length of the finite set of intervals making up $\{x:(x, u) \in$ $\left.\bigcup_{j=1}^{N} R_{j}\right\}$ is strictly less than $100 \varepsilon$. Then $\mathcal{P} \backslash \mathcal{P}(v, \varepsilon)$ is closed and nowhere dense in $(\mathcal{P}, d)$.

Proof. It is easy to check that $\mathcal{P}(v, \varepsilon)$ is open. (If $\eta>0$ is sufficiently small, then setting $R_{j}^{\prime}=R_{j}+[-\eta, \eta]^{2}$ it remains true that the length of the finite unions of intervals $\left\{x:(x, y) \in \bigcup_{j=1}^{N} R_{j}^{\prime}\right\}$ is strictly less than $100 \varepsilon$ for all $y \in[0,1] \cap[v-\varepsilon, v+\varepsilon]$. If $P \in \mathcal{P}(v, \varepsilon), P^{\prime} \in \mathcal{P}$ and $d\left(P, P^{\prime}\right)<\eta$ then

$$
\left\{x:(x, y) \in P^{\prime}\right\} \subseteq\left\{x:(x, y) \in \bigcup_{j=1}^{N} R_{j}^{\prime}\right\}
$$

and so $P^{\prime} \in \mathcal{P}(v, \varepsilon)$.)

We need to show that $\mathcal{P}(v, \varepsilon)$ is dense. To this end, let us write $l(x, \theta)$ for the line segment through $(x, v)$ which joins a point on the line $y=0$ to a point on the line $y=1$ and which is at angle $\theta$ to the $y$-axis. We start with a bit of technical tidying up. Observe that, if $P \in \mathcal{P}$ and $1>\eta>0$, then writing

$$
\begin{aligned}
P^{\prime}= & \bigcup\{l(x+\eta, \theta): l(x, \theta) \subseteq P \text { and } x \leq 0\} \\
& \cup \bigcup\{l(x-\eta, \theta): l(x, \theta) \subseteq P \text { and } x \geq 0\}
\end{aligned}
$$

we have $P^{\prime} \in \mathcal{P}, d\left(P, P^{\prime}\right) \leq \eta$ and $P^{\prime} \subseteq[-1+\eta, 1-\eta] \times[0,1]$.

Thus, to show that $\mathcal{P}(v, \varepsilon)$ is dense it suffices to show that, given $\delta>0$, $\eta>0$ and $P \in \mathcal{P}$ with $P \subseteq[-1+\eta, 1-\eta] \times[0,1]$, we can find a $P^{\prime} \in \mathcal{P}(y, \varepsilon)$ with $d\left(P, P^{\prime}\right)<\delta$. To this end, note that we can find a $\varrho>0$ such that, 
writing

$$
Q=\bigcup\{l(x, \phi):|\phi-\theta| \leq \varrho \text { and } l(x, \theta) \subseteq P\},
$$

we have $Q \in \mathcal{P}$ and $d(P, Q)<\delta / 2$. We observe that the set of open intervals $(\theta-\varrho, \theta+\varrho)$ with $l(x, \theta) \subseteq P$ is an open cover of $[-\pi / 6, \pi / 6]$ (by condition (ii) of Definition 2.1) and so, by compactness, we can find $x_{1}, \ldots, x_{M}$ and $\theta_{1}, \ldots, \theta_{M}$ such that $l\left(x_{m}, \theta_{m}\right) \subseteq P$ for all $1 \leq m \leq M$ and

$$
\bigcup_{m=1}^{M}\left(\theta_{m}-\varrho, \theta_{m}+\varrho\right) \supseteq[-\pi / 6, \pi / 6] .
$$

We can now find $\varrho_{m}$ and $\varrho_{m}^{\prime}$ such that $\varrho \geq \varrho_{m}, \varrho_{m}^{\prime}>0$ for $1 \leq m \leq M$,

$$
\bigcup_{n=1}^{M}\left(\theta_{m}-\varrho_{m}^{\prime}, \theta_{m}+\varrho_{m}\right) \supseteq[-\pi / 6, \pi / 6] \text { and } \sum_{m=1}^{M}\left(\varrho_{m}+\varrho_{m}^{\prime}\right) \leq \pi \text {. }
$$

Setting

$$
Q^{\prime}=\bigcup_{m=1}^{M}\left\{l\left(x_{m}, \phi\right): \phi \in\left(\theta_{m}-\varrho_{m}^{\prime}, \theta_{m}+\varrho_{m}\right)\right\}
$$

we observe that $Q^{\prime} \subseteq Q$ and $Q^{\prime} \in \mathcal{P}$.

A simple compactness argument shows that we can find $\widetilde{x}_{1}, \ldots, \widetilde{x}_{\widetilde{M}}$ and $\widetilde{\theta}_{1}, \ldots, \widetilde{\theta}_{\widetilde{M}}$ such that $l\left(\widetilde{x}_{m}, \widetilde{\theta}_{m}\right) \subseteq P$ for all $1 \leq m \leq \widetilde{M}$ and, writing

$$
Q^{\prime \prime}=\bigcup_{m=1}^{\widetilde{M}} l\left(\widetilde{x}_{m}, \widetilde{\theta}_{m}\right)
$$

we have $d\left(P, Q^{\prime \prime}\right) \leq \delta / 2$. If we now take $P^{\prime}=Q^{\prime} \cup Q^{\prime \prime}$, then $P^{\prime} \in \mathcal{P}$ and $d\left(P^{\prime}, P\right)<\delta$.

At this point it may be worth the reader's while to sketch $P^{\prime}$. If $1 \geq y+\varepsilon$ the set

$$
P^{\prime} \cap\{(x, v):-1 \leq x \leq 1, v \leq y \leq v+\varepsilon\}
$$

consists of a finite set of lines and a finite set of triangles with vertices on the line $y=v$ and bases on the line $y=v+\varepsilon$ of total length less than $4 \pi \varepsilon$ (it is not necessary to make best estimates here). But it is trivial that a triangle of base $K \varepsilon$ can be covered by a finite collection of rectangles $R_{1}^{\prime}, \ldots, R_{q}^{\prime}$, say, with one side parallel to the base in such a way that the intersection of any line parallel to the base with $\bigcup_{j=1}^{N} R_{j}$ has length at most $K \varepsilon$.

It is thus clear that there exist a finite collection of rectangles $R_{1}, \ldots, R_{N}$ with sides parallel to the axes such that, if $y \in[0,1] \cap[v-\varepsilon, v+\varepsilon]$, then

$$
\{x:(x, y) \in P\} \subseteq\left\{x:(x, y) \in \bigcup_{j=1}^{N} R_{j}\right\}
$$


and the total length of the finite set of intervals making up $\{x:(x, u) \in$ $\left.\bigcup_{j=1}^{N} R_{j}\right\}$ is strictly less than $100 \varepsilon$.

We see that Lemma 2.4 proves a result which is slightly stronger than Theorem 2.3. (Observe that, by Fubini's theorem, every member of $\mathcal{E}$ has Lebesgue measure zero.)

Theorem 2.5. The set $\mathcal{E}$ of $E \in \mathcal{P}$ such that $\{x:(x, u) \in E\}$ has Lebesgue measure zero is of second category in $(\mathcal{P}, d)$.

Proof. Lemma 2.4 tells us that (in the notation of that lemma) $\mathcal{P}(r / n, 1 / n)$ is of second category for all integers $r$ and $n$ with $0 \leq r \leq n$. Thus, setting $\mathcal{P}_{n}=\bigcap_{r=0}^{n} \mathcal{P}(r / n, 1 / n)$, we know that $\mathcal{P}_{n}$ is of second category. By the defining property of $\mathcal{P}(r / n, 1 / n)$, we know that, if $P \in \mathcal{P}_{n}$, then $\{x:(x, u) \in P\}$ has Lebesgue measure strictly less than $100 / n$ for all $u \in[0,1]$.

It follows that $\mathcal{P}_{*}=\bigcap_{n=1}^{\infty} \mathcal{P}_{n}$ is of second category and, if $P \in \mathcal{P}_{*}$, then $\{x:(x, u) \in P\}$ has Lebesgue measure zero for all $u \in[0,1]$. Thus $\mathcal{E} \supseteq \mathcal{P}_{*}$ and $\mathcal{E}$ is of second category.

3. Other generic properties. In Lemma 2.4, we considered $\mathcal{P}(v, \varepsilon)$ with $0 \leq v \leq 1$. This restriction is not very important and by making appropriate modifications (essentially replacing " $100 \varepsilon$ " by " $100(|v|+1) \varepsilon "$ ) we obtain the following result.

Theorem 3.1. If $P \in \mathcal{P}$, let us write $\widehat{P}$ for the union of all lines passing through $[-1,1] \times\{0\}$ and $[-1,1] \times\{1\}$ whose intersection with $[-1,1] \times[0,1]$ lies in $P$. The set of $P \in \mathcal{P}$ such that $\{x:(x, y) \in \widehat{P}\}$ has Lebesgue measure zero for all $y \in \mathbb{R}$ is of second category in $(\mathcal{P}, d)$.

All our results can be extended in this way but we shall stick to considering $P$ rather than $\widehat{P}$.

Here is another modification of Lemma 2.4.

LEMMA 3.2. Let $h:[0, \infty) \rightarrow[0, \infty)$ be a strictly increasing function with $h(0)=0$. If $v \in[0,1]$ and $\varepsilon>0$ write $\mathcal{Q}(v, \varepsilon)$ for the set of $Q \in \mathcal{P}$ with the following property. There exist $\eta>0, \kappa>0$, an integer $M \geq 1$ and points $x_{j} \in[-1,1]$ such that, writing

$$
R_{j}=\left(x_{j}-\kappa, x_{j}+\kappa\right) \times(v-\eta, v+\eta),
$$

we have

$$
\bigcup_{j=1}^{M} R_{j} \supseteq Q \cap([-1,1] \times(y-\eta, y+\eta))
$$

and $M h(2 \eta)<\varepsilon$. Then $\mathcal{P} \backslash \mathcal{Q}(v, \varepsilon)$ is closed and nowhere dense in $(\mathcal{P}, d)$. 
Proof. The proof is a rerun with modifications of the proof of Lemma 2.4. It is easy to check that $\mathcal{Q}(v, \varepsilon)$ is open.

Now suppose we are given a $\delta>0$ and $P \in \mathcal{P}$. Exactly as in the proof of Lemma 2.4, we can find a $Q \in \mathcal{P}$ with $d(P, Q)<\delta$ having the following property. There exists an integer $M \geq 1$ and points $x_{j} \in[-1,1]$ such that $Q$ is the union of (possibly degenerate) triangles $T_{j}$ with vertex $x_{j}$ and base a subset of $[-1,1] \times\{0\}$ and triangles $T_{j}^{\prime}$ with vertex $x_{j}$ and base a subset of $[-1,1] \times\{1\}[1 \leq j \leq M]$. (In the special case $v=0$ we take $T_{j}=\left\{\left(x_{j}, 0\right)\right\}$. In the special case $v=1$ we take $T_{j}=\left\{\left(x_{j}, 1\right)\right\}$.) Provided we take $\kappa$ small enough (depending on $M$ ) and $\eta$ small enough (depending on $v$ and $\kappa$ ), we will have $M h(2 \eta)<\varepsilon$ and

$$
\bigcup_{j=1}^{M} R_{j} \supseteq Q \cap([-1,1] \times(y-\eta, y+\eta)) .
$$

Thus $Q \in \mathcal{Q}(v, \varepsilon)$ and we have shown that $\mathcal{P} \backslash \mathcal{Q}(v, \varepsilon)$ is nowhere dense.

Lemma 3.2 immediately yields the following corollary.

Lemma 3.3. Let $h:[0, \infty) \rightarrow[0, \infty)$ be a strictly increasing function with $h(0)=0$ and let $E$ be a countable subset of $[0,1]$. Write $\mathcal{Q}_{E}$ for the set of $Q \in \mathcal{P}$ with the following property. Given any $y \in E$ and any integer $p \geq 1$ there exist $\eta>0, \kappa>0$, an integer $M \geq 1$ and points $x_{j} \in[-1,1]$ such that, writing

$$
R_{j}=\left(x_{j}-\kappa, x_{j}+\kappa\right) \times(v-\eta, v+\eta),
$$

we have

$$
\bigcup_{j=1}^{M} R_{j} \supseteq Q \cap([-1,1] \times(y-\eta, y+\eta))
$$

and $M h(2 \eta)<1 / p$. Then $\mathcal{Q}_{E}$ is of second category in $(\mathcal{P}, d)$.

We now follow a traditional path from the condensation of singularities.

Lemma 3.4. Let $h:[0, \infty) \rightarrow[0, \infty)$ be a strictly increasing function with $h(0)=0$ and let $E$ be a countable dense subset of $[0,1]$. Suppose that $\mathcal{Q}_{E}$ is as in Lemma 3.3 and $Q \in \mathcal{Q}_{E}$.

(i) If $n \geq 1$, write $Y_{Q, n}$ for the set of $y \in[0,1]$ with the following property. There exist $\eta>0, \kappa>0$, an integer $M \geq 1$ and points $x_{j} \in[-1,1]$ such that, writing

$$
R_{j}=\left(x_{j}-\kappa, x_{j}+\kappa\right) \times(v-\eta, v+\eta),
$$

we have

$$
\bigcup_{j=1}^{M} R_{j} \supseteq Q \cap([-1,1] \times(y-\eta, y+\eta))
$$


and $M h(2 \eta)<1 / n$. Then $Y_{Q, n}$ is of second category in $[0,1]$ with the usual Euclidean metric.

(ii) Write $Y_{Q}^{*}$ for the set of $y \in[0,1]$ with the following property. Given any $\varepsilon>0$ there exist $\eta>0, \kappa>0$, an integer $M \geq 1$ and points $x_{j} \in[-1,1]$ such that, writing

$$
R_{j}=\left(x_{j}-\kappa, x_{j}+\kappa\right) \times(v-\eta, v+\eta),
$$

we have

$$
\bigcup_{j=1}^{M} R_{j} \supseteq Q \cap([-1,1] \times(y-\eta, y+\eta))
$$

and $M h(2 \eta)<\varepsilon$. Then $Y_{Q}^{*}$ is of second category in $[0,1]$ with the usual Euclidean metric.

Proof. (i) It is easy to check that $Y_{Q, n}$ is open. Since $E \subseteq Y_{Q, n},[0,1] \backslash Y_{Q, n}$ is nowhere dense.

(ii) Observe that $Y_{Q}^{*}=\bigcap_{n=1}^{\infty} Y_{Q, n}$.

Lemma 3.4 has a trivial corollary.

LEMMA 3.5. Let $h:[0, \infty) \rightarrow[0, \infty)$ be a strictly increasing function with $h(0)=0$ and let $E$ be a countable dense subset of $[0,1]$. Suppose $\mathcal{Q}_{E}$ is as in Lemma 3.3 and $Q \in \mathcal{Q}_{E}$. Write $Y_{Q}$ for the set of $y \in[0,1]$ with the following property. Given any integer $p \geq 1$, there exist $\kappa>0$, an integer $M \geq 1$ and points $x_{j} \in[-1,1]$ such that

$$
\bigcup_{j=1}^{M}\left(\left(x_{j}-\kappa, x_{j}+\kappa\right) \times\{y\}\right) \supseteq Q \cap([-1,1] \times\{y\})
$$

and $M h(2 \eta)<1 / p$. Then $Y_{Q}$ is of second category in $[0,1]$ with the usual Euclidean metric.

Proof. With the notation of Lemma 3.4, $Y_{Q} \subseteq X_{Q}$.

Recall that, if $h:[0, \infty) \rightarrow[0, \infty)$ is a strictly increasing function with $h(0)=0$, then we say that a set $A$ in $\mathbb{R}^{n}$ has zero Hausdorff $h$-measure if, given $\varepsilon>0$, we can find balls $B_{k}$ of radius $r_{k}$ such that $\bigcup_{k=1}^{\infty} B_{k} \supseteq A$ and $\sum_{k=1}^{\infty} h\left(r_{k}\right)<\varepsilon$. Lemmas 3.3 and 3.5 thus imply the following theorem.

THEOREM 3.6. Let $h:[0, \infty) \rightarrow[0, \infty)$ be a strictly increasing function with $h(0)=0$. Then there exists a set $\mathcal{Q}$ of second category in $(\mathcal{P}, d)$ such that, if $Q \in \mathcal{Q}$, then $\{x:(x, y) \in Q\}$ has zero Hausdorff $h$-measure for all $y$ in a set of second category in $[0,1]$.

It is well known (see essentially [4, Proposition 12.2]) that a compact set $P$ which contains line segments of length 1 at all angles $\theta$ to the $y$-axis with $|\theta| \leq \pi / 6$ cannot have zero Hausdorff $h$-measure for $h(t)=t^{\alpha}$ with $\alpha<2$ and thus (see [4, Theorem 8.2]) there is a subset $E$ of $\mathbb{R}$ of positive Lebesgue 
measure such that, if $k(t)=t^{\beta}$ with $\beta<1$ and $y \in E$, then $\{x:(x, y) \in P\}$ does not have zero Hausdorff $k$-measure.

If $x_{1} \neq x_{2}$ and $y_{1} \neq y_{2}$, consider the function

$$
f(t)=\frac{x_{1}+t y_{1}}{x_{2}+t y_{2}} .
$$

By applying the intermediate value theorem, we see that $f$ is rational on a dense subset $E$ of $\mathbb{R}$. Thus, if $t \in E$, the equation

$$
n_{1}\left(x_{1}+t y_{1}\right)+n_{2}\left(x_{2}+t y_{2}\right)=0
$$

has non-trivial integer solutions in $n_{1}$ and $n_{2}$. This shows that a Besicovitch type set must be rich in arithmetical relations. We shall show that (quasi-all) sets in $\mathcal{P}$ have cross sections without such relations.

We need two definitions to make things precise.

Definition 3.7. If $E$ is a non-empty subset of $[-1,1]$, we say that $E$ is independent if, whenever $M \geq 1$ and $x_{1}, \ldots, x_{M}$ are distinct elements of $E$, the equation

$$
\sum_{j=1}^{M} n_{j} x_{j}=0
$$

has no non-trivial integer solutions in $n_{j}$.

Let us write $\chi_{m}(t)=\exp i m t$.

Definition 3.8. We say that a non-empty closed subset $E$ of $[-1,1]$ is Kronecker if, whenever $f:[-1,1] \rightarrow \mathbb{C}$ is a continuous function with $|f(t)|=1$ for all $t \in[-1,1]$ and $\varepsilon>0$, we can find an integer $m$ such that $\left|f(t)-\chi_{m}(t)\right| \leq \varepsilon$ for all $t \in E$.

If $\sum_{j=1}^{M} n_{j} x_{j}=0$ then $\prod_{j=1}^{M} \chi\left(x_{j}\right)^{n_{j}}$ so a Kronecker set must be independent.

We shall prove the following theorem.

TheOREM 3.9. There exists a set $\mathcal{Q}$ of second category in $(\mathcal{P}, d)$ such that, if $Q \in \mathcal{Q}$, then $\{x:(x, y) \in Q\}$ is a Kronecker set for all $y$ in a set of second category in $[0,1]$.

As might be expected, Theorem 3.9 follows from a variation on Lemma 3.2 .

Lemma 3.10. Let $f:[-1,1] \rightarrow \mathbb{C}$ be a continuous function with $|f(t)|=1$ for all $t \in[-1,1]$. If $v \in[0,1]$ and $\varepsilon>0$ write $\mathcal{Q}(v, \varepsilon)$ for the set of $Q \in \mathcal{P}$ with the following property. There exist $\eta>0$ and an integer $m \geq 1$ such that

$$
\left|f(x)-\chi_{m}(x)\right|<\varepsilon \quad \text { for all }(x, y) \in Q \text { with }|y-v|<\eta .
$$

Then $\mathcal{P} \backslash \mathcal{Q}(v, \varepsilon)$ is closed and nowhere dense in $(\mathcal{P}, d)$. 
Proof. It is easy to check that $\mathcal{Q}(v, \varepsilon)$ is open.

Now suppose we are given a $\delta$ with $1>\delta>0$ and $P \in \mathcal{P}$. By making simple changes in the proof of Lemma 2.4 we can find a $Q \in \mathcal{P}$ with $d(P, Q)<\delta / 2$ having the following property. There exists an integer $M \geq 1$, a real number $\nu>0$ and points $x_{j} \in[-1+\nu, 1-\nu]$ such that $Q$ is the union of (possibly degenerate) triangles $T_{j}$ with vertex $x_{j}$ and base a subset of $[-1+\nu, 1-\nu] \times\{0\}$ and triangles $T_{j}^{\prime}$ with vertex $x_{j}$ and base a subset of $[-1+\nu, 1-\nu] \times\{1\}[1 \leq j \leq M]$. (In the special case $v=0$ we take $T_{j}=\left\{\left(x_{j}, 0\right)\right\}$. In the special case $v=1$ we take $T_{j}=\left\{\left(x_{j}, 1\right)\right\}$.)

By uniform continuity, we can find a $\delta^{\prime}$ with $\min (\delta, \nu) / 4>\delta^{\prime}>0$ and $|f(s)-f(t)|<1 / 2$ for all $|s-t|<\delta^{\prime}$ and $s, t \in[-1,1]$. Choose an integer $m \geq 100 / \delta^{\prime}$. By the intermediate value theorem we can find $\widetilde{x}_{j}$ such that

$$
\chi_{m}\left(\widetilde{x}_{j}\right)=f\left(\widetilde{x}_{j}\right) \text { and }\left|\widetilde{x}_{j}-x_{j}\right|<\delta^{\prime}
$$

for all $1 \leq j \leq M$. Now set

$$
\widetilde{T}_{j}=T_{j}+\left(\widetilde{x}_{j}-x_{j}, 0\right)
$$

for $1 \leq j \leq M$ and take $\widetilde{Q}=\bigcup_{j=1}^{M} \widetilde{T}_{j}$. We have $\widetilde{Q} \in \mathcal{P}$ and $d(P, \widetilde{Q})<\delta$. Finally, by uniform continuity again, there exists an $\eta>0$ such that

$$
\left|f(x)-\chi_{m}(x)\right|<\varepsilon \quad \text { for all }(x, y) \in \widetilde{Q} \text { with }|y-v|<\eta .
$$

Thus $\mathcal{P} \backslash \mathcal{Q}(v, \varepsilon)$ is nowhere dense in $(\mathcal{P}, d)$.

Write $S([-1,1])$ for the set of continuous functions $f:[-1,1] \rightarrow \mathbb{C}$ with $|f(t)|=1$ for all $t \in[-1,1]$. Using uniform continuity it is easy to check that there exists a sequence $f_{n} \in S([-1,1])$ which is uniformly dense in $S([-1,1])$. The following is an immediate corollary of Lemma 3.10.

Lemma 3.11. Let $E$ be a countable subset of $[0,1]$. Write $\mathcal{Q}_{E}$ for the set of $Q \in \mathcal{P}$ with the following property. Given any $y \in E$ and any integers $p, q \geq 1$ there exists an $\eta>0$ and an integer $m \geq 1$ such that

$$
\left|f_{q}(x)-\chi_{m}(x)\right|<1 / p \quad \text { for all }(x, y) \in \widetilde{Q} \text { with }|y-v|<\eta \text {. }
$$

Then $\mathcal{Q}_{E}$ is of second category in $(\mathcal{P}, d)$.

The proof of Theorem 3.9 from Lemma 3.11 now follows the pattern of the proof of Lemma 3.5 .

4. An isotropic Besicovitch set. The object of this final section is to prove Theorem 1.1 in the case $n=2$ (the proof for higher dimensions is essentially identical). Throughout this section $a$ will be a fixed number with $1>a>0$ and constants $A_{j}$ will depend on the value of $a$ chosen. We work in $\mathbb{R}^{2}$. We write $D$ for the disc with centre 0 and radius 1 . We shall take "chord" to mean a line segment joining points on the boundary of $D$.

We follow the arguments of Section 2 closely. We study the following set. 
Definition 4.1. $\mathcal{P}$ is the collection of all closed subsets $P$ of the disc $D$ such that

(i) $P$ is the union of a collection $L_{P}$ of chords of length at least $2-a$.

(ii) If $\mathbf{u}$ is a unit vector we can find an $l \in L_{P}$ which is parallel to $\mathbf{u}$.

Lemma 4.2. If $d$ is the Hausdorff metric on $\mathcal{P}$, then $(\mathcal{P}, d)$ is complete.

Proof. Similar to Lemma 2.2.

We shall need to know that certain sets are closed. The following results are typical.

Lemma 4.3. (i) Suppose $P \in \mathcal{P}$ and $0<\theta<\pi / 2$. Write $E_{\theta}$ for the union of all chords $l \subseteq P$ of length at least $2-a$, making an angle $\phi$ with the vertical such that $|\phi| \leq \theta$. Then $E_{\theta}$ is closed.

(ii) Suppose $P \in \mathcal{P}$ and $1 \geq c(1) \geq c(2) \geq 2-a$. Write $F_{c(1), c(2)}$ for the union of all chords $l \subseteq P$ of length $|l|$ such that $c(1) \geq|l| \geq c(2)$. Then $F_{c(1), c(2)}$ is closed.

Proof. (i) Let $\mathbf{z}_{j} \in E_{\theta}$ and $\left\|\mathbf{z}_{j}-\mathbf{z}\right\| \rightarrow 0$ as $j \rightarrow \infty$. Then $\mathbf{z}_{j} \in l_{j}$ where $l_{j}$ is a chord of length at least $2-a$, joining points $\mathbf{a}_{j}$ and $\mathbf{b}_{j}$ on the boundary of $D$ and making an angle $\phi_{j}$ with the vertical such $\left|\phi_{j}\right| \leq \theta$. By using compactness twice, we can find a sequence $n_{j} \rightarrow \infty$ and points $\mathbf{a}$ and $\mathbf{b}$ on the boundary of $D$ such that $\left\|\mathbf{a}_{n(j)}-\mathbf{a}\right\| \rightarrow 0$ and $\left\|\mathbf{b}_{n(j)}-\mathbf{b}\right\| \rightarrow 0$ as $j \rightarrow \infty$. Since $P$ is closed, the chord $l$ joining $\mathbf{a}$ and $\mathbf{b}$ is a subset of $P$. It is easy to check that $l$ has length at least $2-a$, that $l$ makes an angle $\phi$ with the vertical such that $|\phi| \leq \theta$ and that $\mathbf{z} \in l$. Thus $\mathbf{z} \in E_{\theta}$ and $E_{\theta}$ is closed.

(ii) Similar.

If $\kappa>0$ we write $D_{\kappa}$ for the closed disc with centre the origin and radius $\kappa$.

Lemma 4.4. There is a constant $A_{1}$, depending only on a, such that the complement $\mathcal{P} \backslash \mathcal{P}(v, \mathbf{u}, \varepsilon)$ of the set $\mathcal{P}(v, \mathbf{u}, \varepsilon)$ defined in the next paragraph is closed and nowhere dense in $(\mathcal{P}, d)$.

Let $v \in[0,1], \mathbf{u}$ be a unit vector and $1>\varepsilon>0$. Let $\mathbf{u}^{\perp}$ be a unit vector perpendicular to $\mathbf{u}$. We define $\mathcal{P}(v, \mathbf{u}, \varepsilon)$ to be the collection of $P \in \mathcal{P}$ with the following property. There exists a $\kappa$ with $\varepsilon \geq \kappa>0$ such that the following is true. Write $E(\mathbf{u}, \varepsilon-\kappa)$ for the union of all chords $l \subseteq P$ which are of length at least $2-a$ and make an angle $\phi$ with $\mathbf{u}^{\perp}$ such that $|\phi| \leq \pi / 2+\kappa-\varepsilon$. There exists a set $H^{\prime}$ such that writing $H=H^{\prime}+D_{\kappa}$, if $l^{*}$ is any chord with

$$
l^{*} \subseteq\left\{x \mathbf{u}+v \mathbf{u}^{\perp}: x \in \mathbb{R},|v-y|<\varepsilon^{2}\right\},
$$

then $E(\mathbf{u}, \varepsilon-\kappa) \cap l$ has linear Lebesgue measure strictly less than $A_{1} \varepsilon$. 
Proof. There is no loss in generality in taking $\mathbf{u}=(1,0), \mathbf{u}^{\perp}=(0,1)$ and $v \geq 0$. We may also assume that $10^{-3}>\varepsilon$. We try to follow the pattern of the proof of Lemma 2.4. It is easy to check that $\mathcal{P}(v, \mathbf{u}, \varepsilon)$ is open. We need to show that $\mathcal{P}(v, \mathbf{u}, \varepsilon)$ is dense.

We start with a bit of technical tidying up. Suppose $P \in \mathcal{P}$ and $a / 2>$ $\eta>0$. Let $F_{1}$ be the set of chords $l \subseteq P$ with length $|l|$ satisfying $2 \geq|l| \geq 2-a / 2$ and $F_{2}$ be the set of chords $l \subseteq P$ with length $|l|$ satisfying $2-a / 2 \geq|l| \geq 2-a$. Let $\widetilde{F}_{1}$ be the set of chords $\widetilde{l}$ for which there exists an $l \in F_{1}$ such that $\widetilde{l}$ is the closest chord parallel to $l$ which has length $|l|-\eta$. Let $\widetilde{F}_{2}$ be the set of chords $\widetilde{l}$ for which there exists an $l \in F_{2}$ such that $\widetilde{l}$ is the closest chord parallel to $l$ which has length $|l|+\eta$. If we write $P^{\prime}$ for the closure of the union of chords belonging to $\widetilde{F}_{1} \cup \widetilde{F}_{2}$ then $P^{\prime} \in \mathcal{P}$, $d\left(P, P^{\prime}\right) \leq \eta$ and $P^{\prime}$ is the union of chords $l$ with $2-a+\eta \leq|l| \leq 2-\eta$.

Thus to show that $\mathcal{P}(v, \mathbf{u}, \varepsilon)$ is dense it suffices to show that, given $\delta>0$, $\eta>0$ and $P \in \mathcal{P}$ which is the union of chords $l$ with $2-a+\eta \leq|l| \leq 2-\eta$, we can find a $P^{\prime} \in \mathcal{P}(y, \mathbf{u}, \varepsilon)$ with $d\left(P, P^{\prime}\right)<\delta$. To this end write $\widehat{E}(\mathbf{u}, \varepsilon / 2)$ for the set of lines whose intersections with $D$ belong to $E(\mathbf{u}, \varepsilon / 2)$ and set $F(\mathbf{u}, \varepsilon / 2)=\bigcup E(\mathbf{u}, \varepsilon / 2)$. Simple trigonometry shows that there is a constant $A_{2}$, depending on $a$ alone, such that every line in $\widehat{E}(\mathbf{u}, \varepsilon / 2)$ must intersect the line $y=v$ at a point $(x, v)$ with $|x| \leq A_{2}$.

Let us write $\widehat{l}(x, \theta)$ for the line through $(x, v)$ at angle $\theta$ to the $y$-axis and $l(x, \theta)=\widehat{l}(x, \theta) \cap D$. Simple trigonometry shows that there exists a $\varrho$ with $\varepsilon / 4>\varrho>0$ such that, if $|x| \leq A_{2},|\theta| \leq \pi / 2-\varepsilon / 2$ and $l(x, \theta) \subseteq P$ then $2-\eta / 2 \geq|l(x, \theta+\tau)| \geq 2-a+\eta / 2$ and $d(l(x, \theta+\tau), l(x, \theta)) \leq \delta / 4$ whenever $|\tau| \leq \varrho$.

Just as in the proof of Lemma 2.4, we observe that the set of open intervals $(\theta-\varrho, \theta+\varrho)$ with $l(x, \theta) \subseteq P$ and $|x| \leq A_{2}$ is an open cover of $[\varepsilon / 2-\pi / 2, \pi / 2-\varepsilon / 2]$ and so, by compactness, we can find $x_{1}, \ldots, x_{M}$ and $\theta_{1}, \ldots, \theta_{M}$ such that $l\left(x_{m}, \theta_{m}\right) \subseteq P$ for all $1 \leq m \leq M$ and

$$
\bigcup_{m=1}^{M}\left(\theta_{m}-\varrho, \theta_{m}+\varrho\right) \supseteq[\varepsilon / 2-\pi / 2, \pi / 2-\varepsilon / 2] .
$$

We can now find $\varrho_{m}$ and $\varrho_{m}^{\prime}$ such that $\varrho \geq \varrho_{m}, \varrho_{m}^{\prime}>0$ for $1 \leq m \leq M$,

$$
\bigcup_{n=1}^{M}\left(\theta_{m}-\varrho_{m}^{\prime}, \theta_{m}+\varrho_{m}\right) \supseteq[\varepsilon / 2-\pi / 2, \pi / 2-\varepsilon / 2], \quad \sum_{m=1}^{M}\left(\varrho_{m}+\varrho_{m}^{\prime}\right) \leq \pi .
$$

Setting

$$
Q^{\prime}=\bigcup\left\{l\left(x_{m}, \phi\right): \phi \in\left(\theta_{m}-\varrho_{m}^{\prime}, \theta_{m}+\varrho_{m}\right), 1 \leq m \leq M\right\}
$$

we observe that $Q^{\prime} \cup E(\mathbf{u}, 3 \varepsilon / 4) \in \mathcal{P}$ and $d\left(Q^{\prime} \cup F(\mathbf{u}, \varepsilon / 2), F(\mathbf{u}, \varepsilon / 2)\right) \leq \delta / 2$. 
A simple compactness argument shows that we can find $\widetilde{x}_{1}, \ldots, \widetilde{x}_{\widetilde{M}}$ and $\widetilde{\theta}_{1}, \ldots, \widetilde{\theta}_{\widetilde{M}}$ such that $l\left(\widetilde{x}_{m}, \widetilde{\theta}_{m}\right) \subseteq F(\mathbf{u}, \varepsilon / 2)$ for all $1 \leq m \leq \widetilde{M}$ and, writing

$$
Q^{\prime \prime}=\bigcup_{m=1}^{\widetilde{M}} l\left(\widetilde{x}_{m}, \widetilde{\theta}_{m}\right),
$$

we have $d\left(F(\mathbf{u}, \varepsilon / 2), Q^{\prime \prime}\right) \leq \delta / 2$. If we now take $P^{\prime}=Q^{\prime} \cup Q^{\prime \prime} \cup E(\mathbf{u}, 3 \varepsilon / 4)$ then $P^{\prime} \in \mathcal{P}$ and $d\left(P^{\prime}, P\right)<\delta$.

We need to show $P^{\prime} \in \mathcal{P}(v, \mathbf{u}, \varepsilon)$. Write $E^{\prime}(\mathbf{u}, 3 \varepsilon / 4)$ for the union of all chords $l \subseteq P$ which are of length at least $2-a$ and make an angle $\phi$ with $\mathbf{u}^{\perp}$ such that $|\phi| \leq \pi / 2-3 \varepsilon / 4$. Then

$$
\begin{aligned}
E(\mathbf{u}, 3 \varepsilon / 4) \cap\left\{x \mathbf{u}+y \mathbf{u}^{\perp}: x \in \mathbb{R}, y \in[0,1] \cap\left[v-\varepsilon^{2}, v+\varepsilon^{2}\right]\right\} & \\
& \subseteq\left\{x \mathbf{u}+y \mathbf{u}^{\perp} \in Q^{\prime} \cup Q^{\prime \prime}: x \in \mathbb{R}, y \in\left[v-\varepsilon^{2}, v+\varepsilon^{2}\right]\right\} .
\end{aligned}
$$

Thus it will follow that $P^{\prime} \in \mathcal{P}(v, \mathbf{u}, \varepsilon)$ if we can show that there exists a constant $A_{1}$ (depending only on $a$ ) such that (provided only that $\kappa$ is small enough), if we write $H^{\prime}=Q+Q^{\prime}$ and

$$
H=H^{\prime}+\{\mathbf{x}:\|\mathbf{x}\|<\kappa\}
$$

and if $l^{*}$ is any chord with

$$
l^{*} \subseteq\left\{x \mathbf{u}+v \mathbf{u}^{\perp}: x \in \mathbb{R},|v-y|<\varepsilon^{2}\right\},
$$

then $E(\mathbf{u}, \varepsilon-\kappa) \cap l^{*}$ has linear Lebesgue measure strictly less than $A_{1} \varepsilon$.

To see this, look at

$$
\begin{aligned}
T_{m}= & \bigcup\left\{l\left(x_{m}, \phi\right): \phi \in\left(\theta_{m}-\varrho_{m}^{\prime}, \theta_{m}+\varrho_{m}\right)\right\} \\
& \cap\left\{(x, y): x \in \mathbb{R}, y \in\left[v-\varepsilon^{2}, v+\varepsilon^{2}\right]\right\} .
\end{aligned}
$$

If $l^{*}$ is as in the previous paragraph, then $l^{*} \cap T_{m}$ is the the base of a triangle of height no greater than $\varepsilon^{2}$ and with base angles at least $\varepsilon / 8$. By simple trigonometry, there exists an $A_{3}$ (depending on $a$ alone) such that $l^{*} \cap T_{m}$ has length less than $A_{3}\left(\varrho_{m}+\varrho_{m}^{\prime}\right)$. A compactness argument (or more trigonometry) shows that (provided only $\kappa$ is small enough) $l^{*} \cap\left(T_{m}+D_{\kappa}\right)$ has length less than $2 A_{3}\left(\varrho_{m}+\varrho_{m}^{\prime}\right)$ for all $l^{*}$ of the kind specified in the previous paragraph. The desired conclusion now follows.

Lemma 4.5. There is a constant $A_{1}$, depending only on a, such that the set $\mathcal{P}(\varepsilon)$ defined in the next paragraph is of second category in $(\mathcal{P}, d)$.

$\mathcal{P}(\varepsilon)$ is the set of $P \in \mathcal{P}$ with the following property. Suppose $l^{*}$ is a chord parallel to a unit vector $\mathbf{w}$. Let $\mathbf{w}^{\perp}$ be a unit vector perpendicular to $\mathbf{w}$ and write $E(\mathbf{w}, \varepsilon / 2)$ for the union of all chords $l \subseteq P$ which are of length at least $2-a$ and make an angle $\phi$ with $\mathbf{u}^{\perp}$ such that $|\phi| \leq \pi / 2-\varepsilon / 2$. Then $E(\mathbf{w}, \varepsilon / 2) \cap l^{*}$ has linear Lebesgue measure strictly less than $A_{1} \varepsilon$. 
Proof. Without loss of generality suppose $10^{-2} \geq \varepsilon$. We can find a finite collection of ordered pairs $\left(v_{m}, \mathbf{u}_{m}\right)$ with $\left|v_{m}\right| \leq 1$ and $\mathbf{u}_{m}$ a unit vector $[1 \leq m \leq M]$ with the following property. Choose unit vectors $\mathbf{u}_{m}^{\perp}$ perpendicular to $\mathbf{u}_{m}$. If $l^{*}$ is any chord then we can find an $m$ with $1 \leq m \leq M$ such that

$$
l^{*} \subseteq\left\{x \mathbf{w}+v \mathbf{w}^{\perp}: x \in \mathbb{R},|v-y| \leq \varepsilon^{2} / 2\right\} .
$$

We use the notation of Lemma 4.4. Set $\mathcal{Q}(\varepsilon)=\bigcup_{m=1}^{M} \mathcal{P}\left(v_{m}, \mathbf{u}_{m}, \varepsilon\right)$. If $P \in \mathcal{Q}(\varepsilon)$ and $l^{*}$ is any chord then, writing $E(\mathbf{w}, \varepsilon)$ for the union of all chords $l \subseteq P$ which are of length at least $2-a$ and make an angle $\psi$ with $l^{*}$ such that $|\psi| \geq \varepsilon$, we know, by the definition of $\mathcal{P}\left(v_{m}, \mathbf{u}_{m}, \varepsilon\right)$ in Lemma 4.4, that $E(\mathbf{w}, \varepsilon / 2) \cap l^{*}$ has linear Lebesgue measure strictly less than $A_{1} \varepsilon$. Thus $\mathcal{Q}(\varepsilon) \subseteq \mathcal{P}(\varepsilon)$. But, by Lemma $4.4, \mathcal{Q}(\varepsilon)$ is of second category, so $\mathcal{P}(\varepsilon)$ is also of second category.

We are now in a position to prove Theorem 1.1. (As stated earlier we just deal with the case $n=2$.) Since $(\mathcal{P}, d)$ is complete, it follows at once from our next result.

TheOREM 4.6. Consider the set $\mathcal{Q} \subset \mathcal{P}$ satisfying the following condition. Let $\mathcal{L}$ be the set of chords $l$ of length at least $2-$ a such that $l \subseteq Q$. Then, if $l^{*}$ is any chord, $\bigcup_{l \in \mathcal{L}, l \neq l^{*}} l \cap l^{*}$ has zero linear Lebesgue measure.

Then $\mathcal{Q}$ is of second category in $(\mathcal{P}, d)$.

Proof. We use the notation of Lemma 4.5. Set $\mathcal{Q}^{\prime}=\bigcap_{j=1}^{\infty} \mathcal{P}(1 / j)$. By Lemma $4.5, \mathcal{Q}^{\prime}$ is of second category in $(\mathcal{P}, d)$. The theorem will thus follow if we can show that $\mathcal{Q} \supseteq \mathcal{Q}^{\prime}$.

To this end, consider any $P \in \mathcal{Q}$ and any chord $l^{*}$ parallel to a unit vector $\mathbf{w}$. Let $\mathbf{w}^{\perp}$ be a unit vector perpendicular to $\mathbf{w}$ and write $E_{j}=$ $E(\mathbf{w}, 1 /(2 j))$ for the union of all chords $l \subseteq P$ which are of length at least $2-a$ and make an angle $\phi$ with $\mathbf{w}^{\perp}$ such that $|\phi| \leq(\pi-1 / j) / 2$. By the definition of $\mathcal{P}(1 / j), E_{j} \cap l^{*}$ has linear Lebesgue measure strictly less than $A_{1} / j$. Now

$$
E_{1} \cap l^{*} \subseteq E_{2} \cap l^{*} \subseteq, \ldots \quad \bigcup_{j=1}^{\infty}\left(E_{j} \cap l^{*}\right)=\bigcup_{l \in \mathcal{L}, l \neq l^{*}} l \cap l^{*},
$$

so, by monotone convergence, $\bigcup_{l \in \mathcal{L}, l \neq l^{*}} l$ has linear Lebesgue measure zero as required.

\section{References}

[1] R. Alexander, Random compact sets related to the Kakeya problem, Proc. Amer. Math. Soc. (2) 53 (1975), 415-419.

[2] A. S. Besicovitch, On Kakeya's problem and a similar one, Math. Z. 27 (1928), 312-320. 
[3] A. S. Besicovitch, On the fundamental geometrical properties of linearly measurable plane sets of points III, Math. Ann. 116 (1939), 349-357.

[4] K. Falconer, Fractal Geometry, Wiley, Chichester, 1990.

[5] F. Hausdorff, Set Theory, 2nd ed. translated from the German by J. R. Aumann et al., Chelsea, New York, 1957.

[6] J.-P. Kahane, Trois notes sur les ensembles parfaits linéaires, Enseign. Math. (2) 15 (1969), 185-192.

[7] E. M. Stein, Harmonic Analysis, Princeton Univ. Press, Princeton, 1993.

DPMMS, Centre for Mathematical Sciences

Clarkson Road

Cambridge, U.K.

E-mail: twk@dpmms.cam.ac.uk

Received October 28, 2002

Revised version March 19, 2003 